\title{
Process Online Monitoring on Cold Extrusion of Internal Thread in High-strength Steel
}

\author{
Hong Miao ${ }^{1}$, Xiang Shan ${ }^{1}$, Shanwen Zhang ${ }^{1}$, Yifu Jin ${ }^{1}$, Dunwen Zuo ${ }^{2}$ \\ ${ }^{1}$ College of Mechanical Engineering, yangzhou University, yangzhou 225000, China; Email: mh0514@163.com, \\ 1256935251@qq.com, *zhangshanwen123@163.com,dyfjin@yzu.edu.cn \\ ${ }^{2}$ Mechanical Engineering Institute, Nanjing University of Aeronautics and Astronautics, Nanjing 210016, China, ei- \\ mitt505@nuaa.edu.cn
}

In order to solve the problems of data collection, data storage, artificial neural networks, real-time display of images, real-time monitoring of current status and recurrence of historical data, online monitoring system of cold extrusion of internal thread process is designed by LabVIEW8.5. The time-domain analysis and time-frequencydomain analysis are carried out for the torque signal, temperature signal, vibration signal, acoustic emission signal and transmitted signal. The research shows that: this online monitoring system can forecast processing status accurately, raise real-time alarm to replace or re-install the thread forming tap, accordingly avoids effectively the wreckage of thread forming tap and the scrap of work pieces; the fault recognition rate reaches $97 \%$. And the practical model of online monitoring system for cold extrusion of internal thread process can be used to monitor working process.

Keywords: Internal thread; Extrusion; Torque; Temperature; Online monitoring

\section{Introduction}

Cold extrusion net shape process of the internal thread becomes a development trend on the anti-fatigue process of the present aircrafts, high-speed trains. The advantage is to keep the cold work hardening status on the surface and subsurface of internal threads. This not only improves the strength and hardness of material, but also increases the fatigue life of internal threads. Traditional cold extrusion process is only suited to the processes on the nonferrous metals with low-intensity, good plasticity and the low carbon steel. However for the processes on the highstrength steel series used for crafts and high-speed trains, the high-strength steel not only has the higher strength, but also has the higher fracture toughness. But the sensibility on the notch is higher at the same time; stress concentration is easily introduced, and is sensitive to the hydrogen brittleness and stress corrosion. Thus, reasonable processes should be conducted to increase machining precision and surface quality in order to obtain higher fatigue resistance. At present, the researches on this filed are all in fledging period and exploration stage with less of relative achievements [1-6].

During the process of cold extrusion of internal thread, how to avoid the wear, damage and fracture of thread forming tap are the technical problems. Thus, there is vital practical significance to execute online monitoring and real-time alarm during the process of cold extrusion of internal thread. This paper solves a sequence of problems with own designed monitoring system by performing signal processing analysis of the torque, the temperature, vibration, transmitted signal, feature extraction and selection, pattern recognition and other areas, to lay the foundation for the development of cold extrusion of internal thread process[7-12].

This paper designs and produces the online monitoring system of cold extrusion of internal thread process. The virtual instrument technology with LabVIEW8.5 is used as platform to realize the functions of data collection, data storage, artificial neural networks, real-time display of images, real-time monitoring of current status and recurrence of historical data; based on the foundation of the time-domain analysis and time-frequency-domain analysis of the torque signal, temperature signal, vibration signal, acoustic emission signal and transmitted signal, multi-sensors data fusion technology with the collaboration of multiple sensors is used to improve the monitoring precision; this online monitoring system can forecast processing status accurately, raise real-time alarm to replace or re-install the thread forming tap, and also avoids effectively the wreckage of thread forming tap and the scrap of work pieces.

\section{Design and development of online monitoring system}

\subsection{Overall design of online monitoring system}

During the process of cold extrusion of high strength internal thread, the changes of process conditions, such as lubricants, spindle speed of machine, diameter of underport of work pieces, extrusion times, have significant influence on the wear, damage, fracture of thread forming tap and the processing quality of work pieces; the measurement of torque, temperature, vibration and sound signals will provide reliable test parameters for the experimental studies. Thus, based on the features of online testing, online monitoring system is designed, and the torque, temperature, vibration and sound are characteristic parameters. Structure diagram of online monitoring system is shown in fig. 1: the system is consisted of sensors, signal modulation circuit, data acquisition card and computer. The physical parameters such as torque, temperature, vibration and sound during the working process are transformed into analog voltage signals via the sensors, the signals are magnified, filtered and transformed into digital signals; then they are input into computer via 
the data acquisition card and the data is displayed, processed and stored.

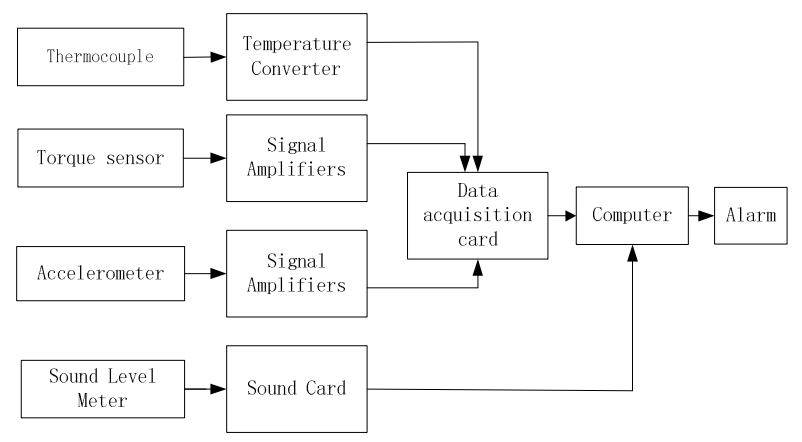

Fig. 1 Structure diagram of basic system composing

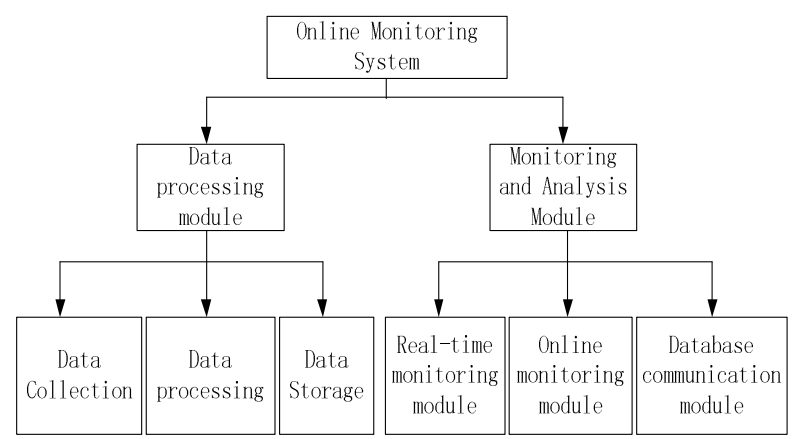

Fig. 2 Schematic diagram of online monitoring system

\subsection{Realization method of hardware for the online monitoring system}

Torque introduced during the process of cold extrusion of internal thread is measured by using the hollow circular shaft type of torque sensor. Resistance strain sensing element is adopted on the torque sensor, and the sensor is consisted of elastic shaft, measurement bridge, instrumentation amplifier and interface circuit. The elastic shaft is auxiliary elastic component; this not only increases the sensitivity of the tangential extrusion torque sensor and also reduces the interference between each other. BF-350HA (23) foil gauge is used as the bridge strain gauge, the normal resistance is $350 \Omega$, and sensitivity coefficient is 2.0; four electric resistance strain gages, which are $45^{\circ}$ angle with the axis, are attached on the elastic shaft with $90^{\circ}$ angle internal.

It is difficult to measure directly the temperature of the extrusion area in case that the deformation area is on the surface of internal bore of the work pieces during the molding phase of internal thread extrusion. Thus, the average temperature of extrusion area is measured after the work pieces are drilled. $\mathrm{K}$ type thermocouple is inserted into the hole of work piece in advanced, and fix it on the three-jaw chuck directly, both positive and negative electrodes of the thermocouple are lead out and connected with XMT-3000 industrial controler/regulator via the current collecting equipment at the foot section of the lathe spindle; the measuring precision is stable, measuring error introduced by temperature excursion and time drift are eliminated, and it has strong anti-jamming ability. In the thermocouple input mode, cold end compensating unit is equipped inside the instrument, and also volatge out port is equipped; temperature measured by thermocouple can be obtained after conversion.

TS152000 piezoelectricity acceleration sensor and TS6100 constant current optimal modulator are used to measure the vibration signals during the working process. During the measurement, the piezoelectricity acceleration sensor should be installed at the top point in the front of the clamping torque device, signal from the sensor during the working process will directly be input in the data acquisition channel of the data acquisition card via the current optimal modulator; correction factor should be determined during the measurement and calculation, and input into the stochastic signal and vibration analysis system software, then the vibration acceleration value of the measuring point will be output via the computer.

AWA5661 type impulse precision sound level meter is used to measure the sound signals during the working process; during the measurement, the sound level meter should be fixed on the board of machine tool, signal from the sensor will directly be input in the data acquisition channel of the data acquisition card, then the instantaneous sound level will be output via the computer.

\subsection{Realization method of hardware for the online monitoring system}

Online monitoring system is an integrated system with monitoring and prediction functions based on database. The database composition is shown in fig. 2; it is separated as two parts: cutting tool information database and working process information database. The cutting tool information database mainly includes the serial number of processing tap, using times, etc. The working process database mainly includes the process status of the cold extrusion of internal thread in different process conditions and the corresponding processed quality of work pieces.

In fig. 2, the online monitoring system mainly includes the data processing module and detection analysis module. The main functions of the data processing module are data collection, data processing, and data storage, etc. The detection analysis module is mainly consisted of real time monitoring module, online monitoring module and database communication module. The real time monitoring module mainly analyzes and judges the stability of process status via the real time monitoring of the torque and temperature during the working process, the module can raise early warnings in the serious situations, like work piece slipping, tap broken, ect, to fulfill the purpose of machine prevention. The online monitoring module is to judge and foresee the wearing status of cutting tools via the comprehensive analysis of the torque, temperature, vibration and sound signals in the previous process after this process is finished. This can provide feasibility prediction if the following process can be continued. The main functions of database communication module is to update the information of the thread forming tap timely after the working process is finished, and to update and supplement the working process information database if necessary. This can obtain better predictive effect[4-5].

Online monitoring system of working process is controlled by system software designed by LabVIEW8.5, the 
front panel of the test system is shown in fig. 3, and it is consisted of four parts: real time display of images, instantaneous display of status parameters, status monitoring indication and the thread forming tap information display.

\section{Selection and extraction of feature vector}

The cold extrusion of internal thread process is a complicated flowing and forming process of metal. In this process, the extrusion torque, average temperature of the extrusion area, vibration of the tap and sound signals of the working process are all changing along with the different working process, but the signal information obtained from the sensor cannot be monitored directly. Due to the amount of the sensor signals is large while the information is quite few, it is difficult to establish the deterministic relationship with the changes of the cold extrusion of internal thread process. Thus the signals must be transformed accordingly to extract the signal features that reflect the changes of working process. This paper uses the multi-sensors data fusion technology to analyze the working process status under different process conditions. This can ensure the cold extrusion of internal thread process in smooth progress, as shown in fig. 4.

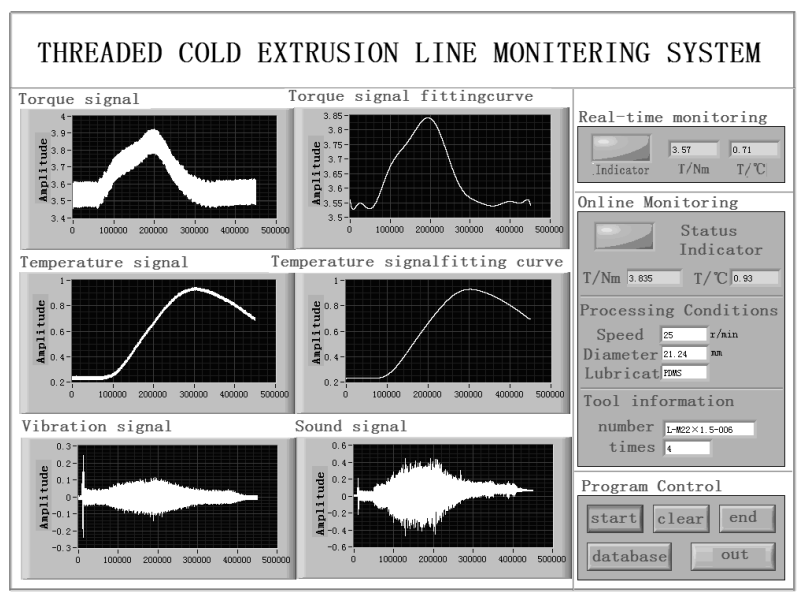

Fig. 3 Front panel of the test systém

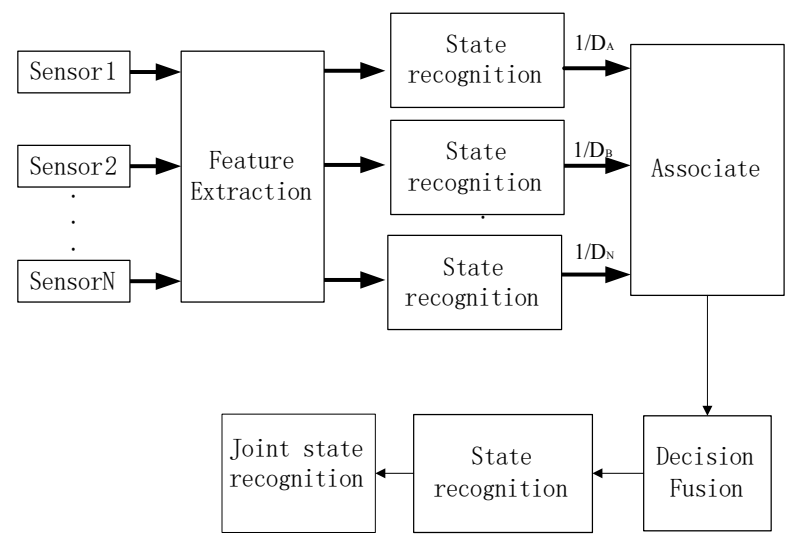

Fig. 4 Decision fusion of sensor data

The monitoring feature of the process changes for cold extrusion of internal thread process is obtained from the time domain and time-frequency domain signals analysis method. The data signals collected during the cold extrusion of internal thread process are classified based on different handling requirements. The extrusion torque and average temperature of extrusion area as changing curve are displayed, and time domain is analyzed and handled. The time domain feature such as the vibration of tap and working process sound should be considered, and the frequency variation law also should be considered, so comprehensive analysis of time domain and time-frequency domain should be conducted for the signals.

\subsection{Signal features of the torque and temperature}

During the cold extrusion of internal thread process, the signal of torque and temperature will be more or less impacted by the external factors. During the actual test process, the $50 \mathrm{~Hz}$ electric supply is the main interference source against the extrusion process signal, and its signal is approximate sinusoidal wave. Least squares curve fit is used to remove the signal interference effectively; the least squares curve fit method is the fitting of a polynomial to minimum the quadratic sum of error, to find out a curve that make the most approach with all data points under the rule of minimum quadratic sum of error.

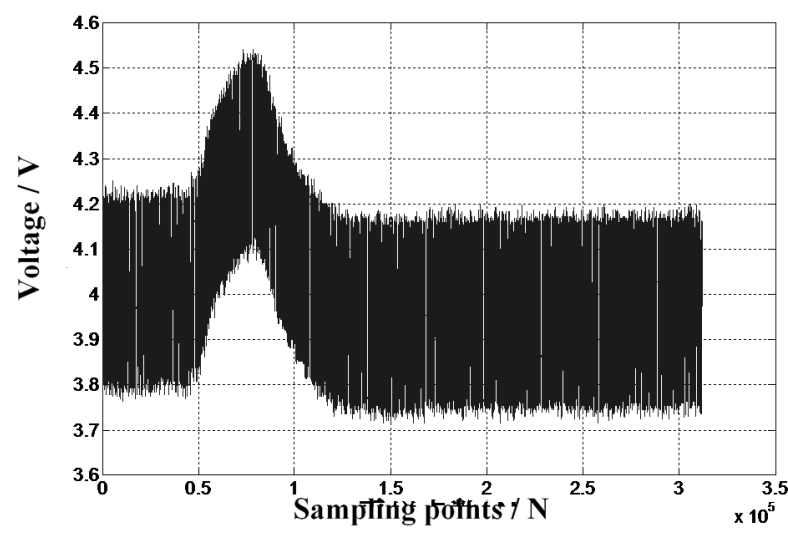

Fig. 5 Time domain waveform figure of the torque original signals

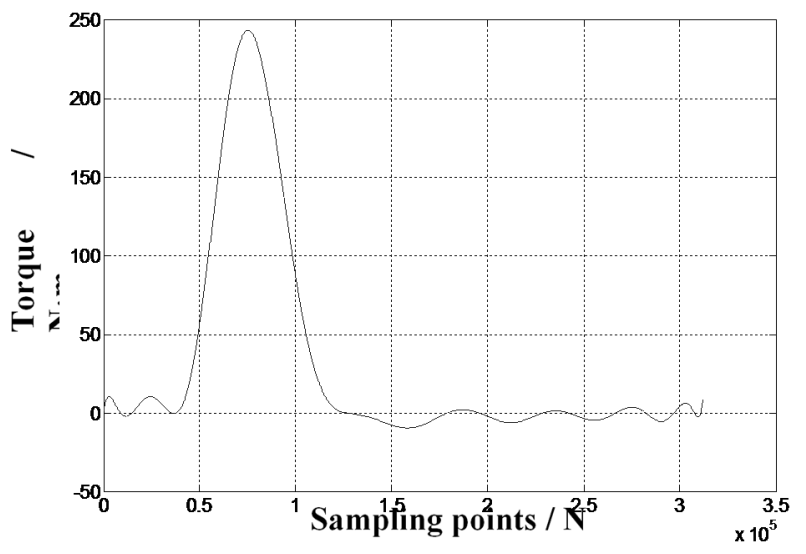

Fig. 6 Torque during cold extrusion of the internal thread process

Those selected signal samples are high strength steel Q460 under the working process, machine rotate speed is $25 \mathrm{r} / \mathrm{min}$ and the length of thread is $20 \mathrm{~mm}$; the extrusion taps are lubricated by PDMS lubrication, the diameter $\mathrm{d} 0$ 
of bottom hole of the sample is $\Phi 21.25 \mathrm{~mm}$. The fig. 5 shows the time domain waveform figure of the original signals, after the original torque signals are handled, the torque in the cold extrusion of internal thread process is obtained, shown in fig. 6 . It can be seen that the torque increases along with the increase of the contacting area between the thread forming tap and the work pieces. Thus torque value can be extracted to be the monitoring feature to identify the working status of the cold extrusion of internal thread process.

The fig. 7 is the time domain waveform figure of temperature original signals during the cold extrusion of internal thread process, the relationship between the output voltage and actual collected temperature can be obtained based on the XMT0-3000 configuration, the temperature curve after de-noising processing is shown in fig. 8 . The change of temperature is similar with the change of the torque, and both of them increase along with the increase of the contacting area between the thread forming tap and the work pieces. This reflects the concerned features during the working processes. Thus temperature value during the extrusion process can be extracted to be the monitoring feature to identify the working status of the cold extrusion of internal thread process.

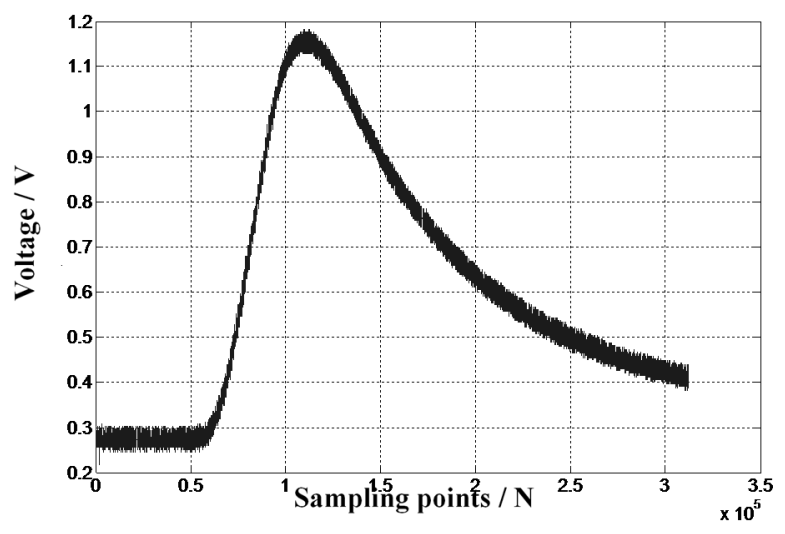

Fig. 7 Time domain waveform figure of the temperature original signals

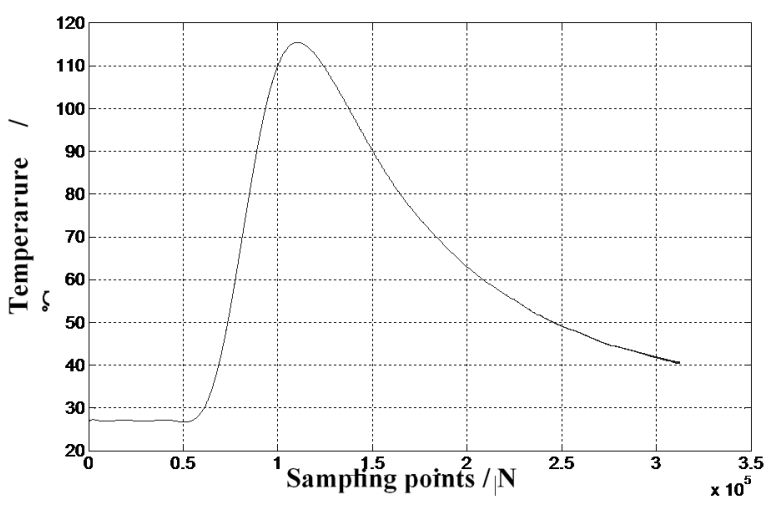

Fig. 8 Temperature during the cold extrusion of internal thread process

\subsection{Signal features of the vibration and sound}

The fig. 9 is the time domain waveform figure of vibration signals of thread forming tap and sound signals of machine tool during the cold extrusion of internal thread process, after the vibration and sound signals are handled, the autocorrelation function is shown in fig. 10. The vibration and sound signals are both stochastic signal during the working process.

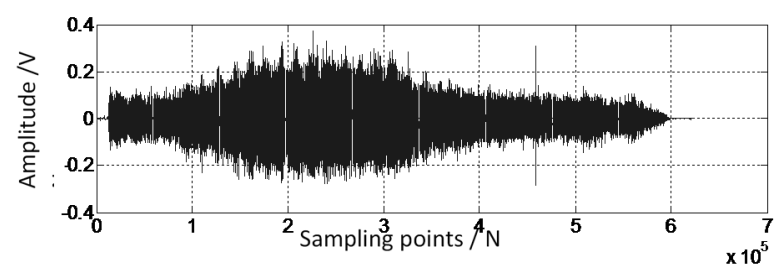

a) Vibration signals of thread forming tap

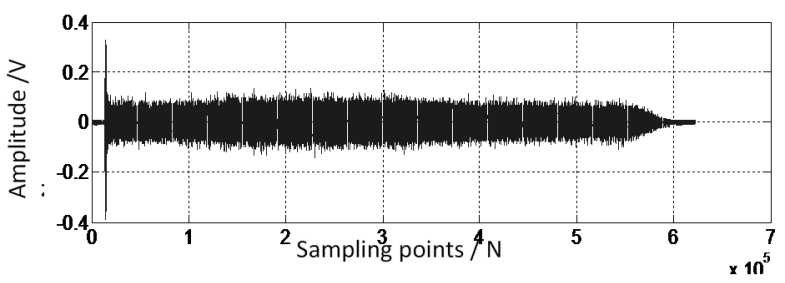

(b) Sound signals of the machine tool

Fig. 9 Time domain waveform figure of vibration signals of thread forming tap and sound signals of macihne tools

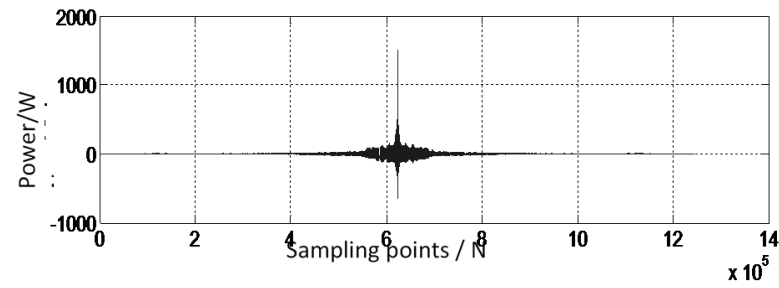

a) Vibration signals of thread forming tap

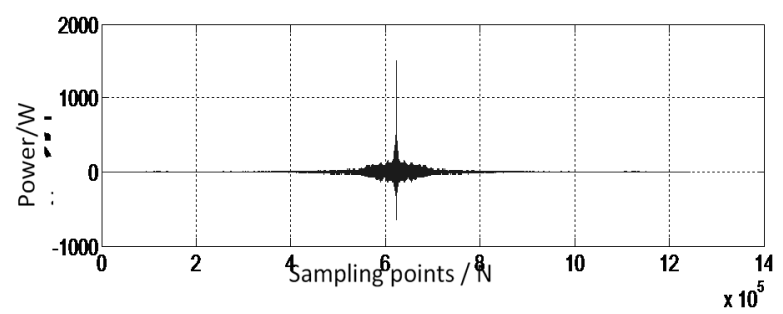

b) Sound signals of the machine tool

Fig. 10 Autocorrelation function of vibration signals of thread forming tap and sound signals of macihne tool

During the cold extrusion of internal thread process, the test results show that under the $5 \mathrm{kHz}$ sampling frequency, the vibration signals of thread forming tap and sound signals of the machine tool are below $1500 \mathrm{~Hz}$ in the normal working situation; when abnormal situation, such as slipping, happens, the high-frequency section energy of signals will increase rapidly, thus the ratio of low and high-frequency section energy reflects effectively the status changes during the working process.

Fig. 11 is the energy ratio of vibration signals of thread forming tap and sound signals of the machine tool under different rotate speed of machine tool. Along with the increase of the rotate speed of machine tools, the energy ratio increases, then reduces. So it can be monitoring feature to identify the working status of the cold extrusion of internal thread process. 


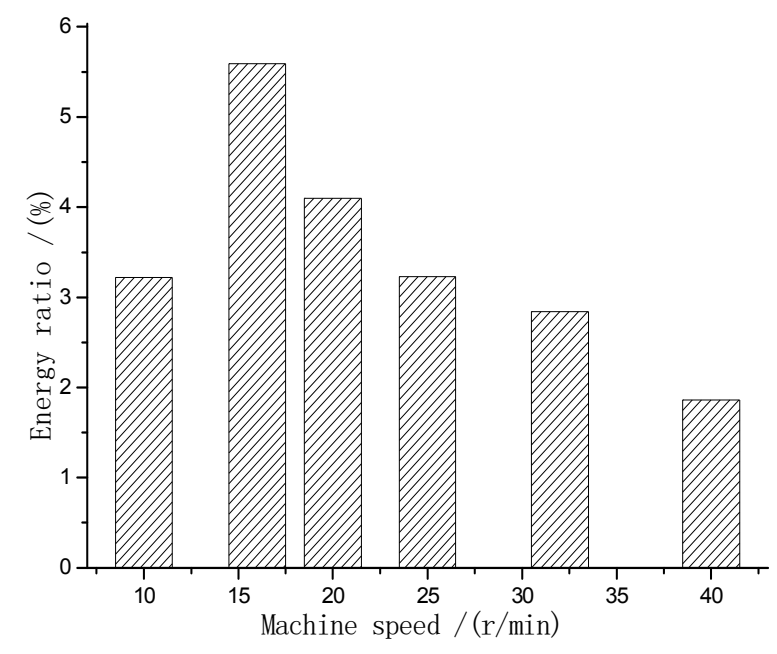

a) Vibration signals of thread forming tap

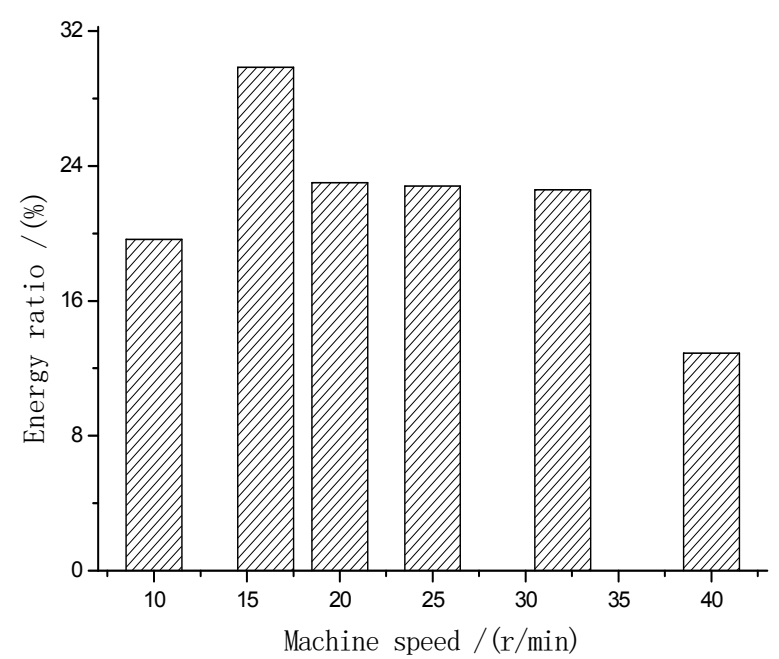

b) Sound signals of the machine tool

Fig. 11 The energy ratio of vibration signals of thread forming tap and sound signals of the machine tool under different rotate speed of machine tool.

Fig. 12 is the high frequency power of vibration signals of thread forming tap and sound signals of the machine tool under different rotate speed of machine tool. Along with the increase of the rotate speed of machine tools, the high frequency power increases, then reduces. So it also can be monitoring feature to identify the working status of the cold extrusion of internal thread process.

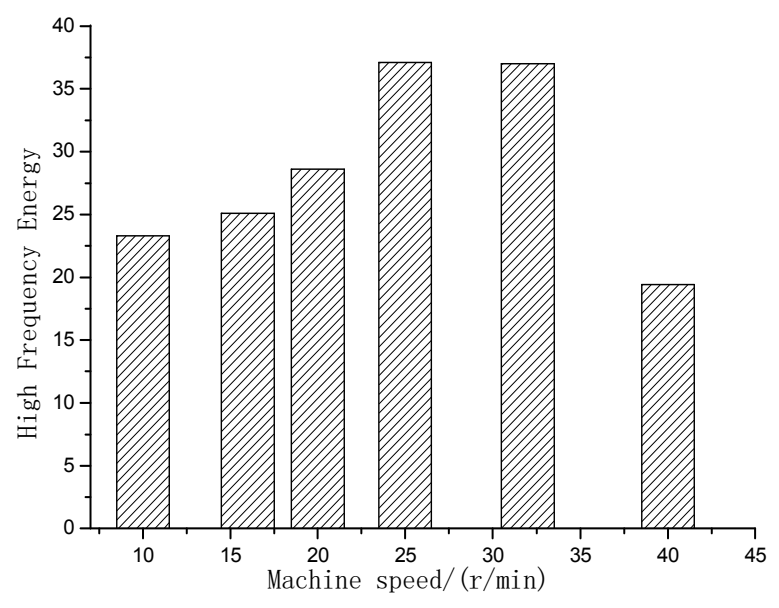

a) Vibration signals of thread forming tap

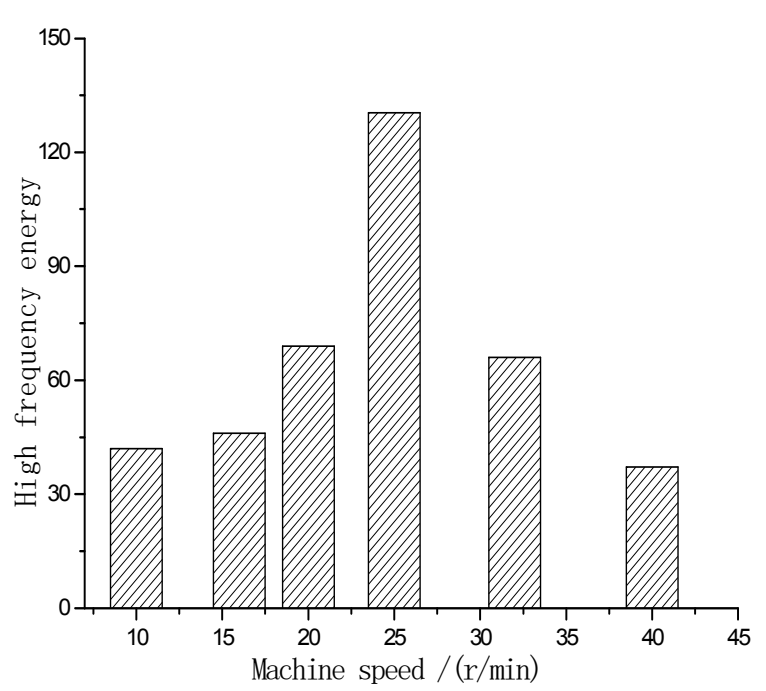

b) Sound signals of the machine tool

Fig. 12 The high frequency power of vibration signals of thread forming tap and sound signals of the machine tool under different rotate speed of machine tool

\section{Analysis of monitoring results}

Online monitoring test is conducted by using the monitoring system, the monitoring results analysis is shown in table 1, there are two thread forming taps damaged without any warning, only one thread forming tap is damaged seriously without any warning. There is no situation that the thread forming tap is slightly worn, damaged and slipping without any warning. The fault recognition rate reaches $97 \%$.

Tab. 1 Monitoring results analysis of thread forming tap worn

\begin{tabular}{|c|c|c|}
\hline Status of working process & Actual amount & Monitoring amount \\
\hline Thread forming tap processed normally & 10 & 10 \\
\hline Thread forming tap slightly worn & 10 & 9 \\
\hline Thread forming tap seriously worn & 10 & 10 \\
\hline Thread forming tap broken & 10 & 10 \\
\hline Work pieces slipping & 10 & 8 \\
\hline
\end{tabular}


After the monitoring system is determined, system test is conducted by using process conditions of the online test. The cold extrusion of internal thread process is conducted on work pieces group by group; the real time changes of the working process are monitored. The test data from the online monitoring system is shown in table 2 to table 5. The wearing status of thread forming tap can be observed by microscope after machine is stopped. The wear mass loss can be determined by the difference value between the sum widths of all edge teeth before and after working process.

Tab. 2 extrusion torque measure data of online monitoring system

\begin{tabular}{|c|c|c|c|c|c|c|c|c|c|c|}
\hline Processing Status & \multicolumn{10}{|c|}{ Extrusion torque (N·m) } \\
\hline Normal processing & 186 & 216 & 201 & 224 & 201 & 201 & 209 & 216 & 231 & 209 \\
\hline Taps mild wear & 238 & 253 & 238 & 246 & 253 & 261 & 253 & 238 & 246 & 253 \\
\hline Taps severe wear & 462 & 499 & 529 & 484 & 507 & 499 & 536 & 522 & 499 & 551 \\
\hline Taps fracture & 894 & 1006 & 946 & 1082 & 944 & 1109 & 916 & 920 & 1047 & 1065 \\
\hline Workpiece slippage & 656 & 678 & 700 & 678 & 715 & 683 & 742 & 779 & 693 & 701 \\
\hline
\end{tabular}

Tab. 3 extrusion temperature measure data of online monitoring system

\begin{tabular}{|c|c|c|c|c|c|c|c|c|c|c|}
\hline Processing Status & \multicolumn{10}{|c|}{ Extrusion temperature $\left({ }^{\circ} \mathbf{C}\right)$} \\
\hline Normal processing & 79 & 82 & 86 & 83 & 83 & 85 & 86 & 85 & 87 & 87 \\
\hline Taps mild wear & 92 & 95 & 94 & 95 & 93 & 94 & 94 & 95 & 97 & 95 \\
\hline Taps severe wear & 98 & 102 & 107 & 100 & 105 & 104 & 110 & 108 & 104 & 110 \\
\hline Taps fracture & 147 & 152 & 138 & 145 & 149 & 144 & 138 & 142 & 144 & 148 \\
\hline Workpiece slippage & 129 & 132 & 140 & 136 & 142 & 125 & 131 & 127 & 125 & 125 \\
\hline
\end{tabular}

Tab. 4 vibration measure data of thread forming tap of online monitoring system

\begin{tabular}{|c|c|c|c|c|c|c|c|c|c|c|}
\hline Processing Status & \multicolumn{1}{|c|}{ Extrusion tap vibration (total energy) } \\
\hline Normal processing & 178 & 175 & 180 & 182 & 175 & 185 & 184 & 182 & 180 & 178 \\
\hline Taps mild wear & 195 & 197 & 200 & 203 & 198 & 207 & 189 & 210 & 205 & 201 \\
\hline Taps severe wear & 225 & 223 & 215 & 220 & 217 & 228 & 219 & 221 & 223 & 231 \\
\hline Taps fracture & 240 & 236 & 227 & 251 & 218 & 226 & 220 & 235 & 241 & 225 \\
\hline Workpiece slippage & 208 & 210 & 205 & 215 & 207 & 213 & 205 & 210 & 212 & 214 \\
\hline Processing Status & & \multicolumn{1}{|c|}{ Extrusion tap vibrations (high frequency) } \\
\hline Normal processing & 21.6 & 21.8 & 21.6 & 21.4 & 21.5 & 21.6 & 21.8 & 21.4 & 21.3 & 21.6 \\
\hline Taps mild wear & 21.9 & 22 & 21.5 & 21.8 & 22.1 & 22.3 & 21.7 & 22.5 & 22.3 & 21.8 \\
\hline Taps severe wear & 27.2 & 26.2 & 26.1 & 28.3 & 27.5 & 27.8 & 26.3 & 28.1 & 27.6 & 29.1 \\
\hline Taps fracture & 27.2 & 24.3 & 22.5 & 29.1 & 26.4 & 25.5 & 26.2 & 24.8 & 26.1 & 23.6 \\
\hline Workpiece slippage & 25.3 & 26 & 24.9 & 25.7 & 25.2 & 25.5 & 26.1 & 25.1 & 25.3 & 25.6 \\
\hline
\end{tabular}

Tab. 5 Online Monitoring System machine sound test data

\begin{tabular}{|c|c|c|c|c|c|c|c|c|c|c|c|}
\hline Processing Status & \multicolumn{10}{|c|}{ Sound Machine (total energy) } \\
\hline Normal processing & 1708 & 1658 & 1835 & 1927 & 1756 & 1870 & 1790 & 1823 & 1760 & 1523 \\
\hline Taps mild wear & 1332 & 1503 & 1221 & 1642 & 1589 & 1613 & 1529 & 1460 & 1439 & 1620 \\
\hline Taps severe wear & 12808 & 14927 & 15303 & 13498 & 11975 & 15630 & 11397 & 13450 & 17932 & 21057 \\
\hline Taps fracture & 20793 & 25340 & 30561 & 31425 & 27431 & 26550 & 35460 & 23521 & 26590 & 24883 \\
\hline Workpiece slippage & 33829 & 37980 & 40294 & 27650 & 35621 & 36435 & 29752 & 34267 & 41535 & 37611 \\
\hline Processing Status & \multicolumn{1}{|c|}{ Sound Machine (energy ratio) } & \multicolumn{4}{|c|}{} \\
\hline Normal processing & 16 & 14.7 & 15 & 16.2 & 12.3 & 11.9 & 13.8 & 15.6 & 14.5 & 13.2 \\
\hline Taps mild wear & 7.5 & 7.2 & 6.5 & 8.4 & 7.9 & 8.7 & 6.8 & 8 & 7.3 & 7.7 \\
\hline Taps severe wear & 2.6 & 3.5 & 2.8 & 3 & 2.7 & 3.4 & 2.6 & 2.8 & 3.5 & 2.8 \\
\hline Taps fracture & 2.2 & 3.4 & 2.5 & 2.3 & 2.4 & 2.4 & 2.7 & 3.3 & 3.5 & 2.1 \\
\hline Workpiece slippage & 3 & 3.2 & 1.9 & 2.3 & 2.7 & 2.5 & 2.1 & 2.4 & 2.9 & 3.1 \\
\hline
\end{tabular}



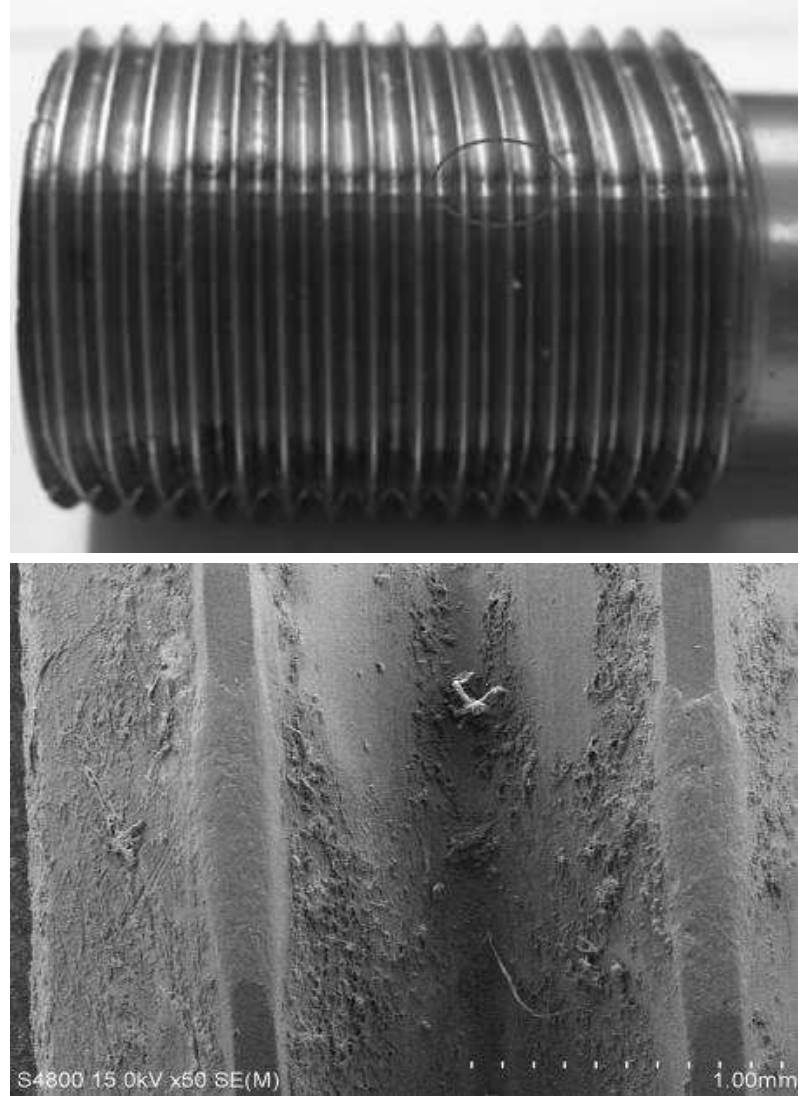

a) Slightly wearing status and partial enlarged drawing of thread forming tap

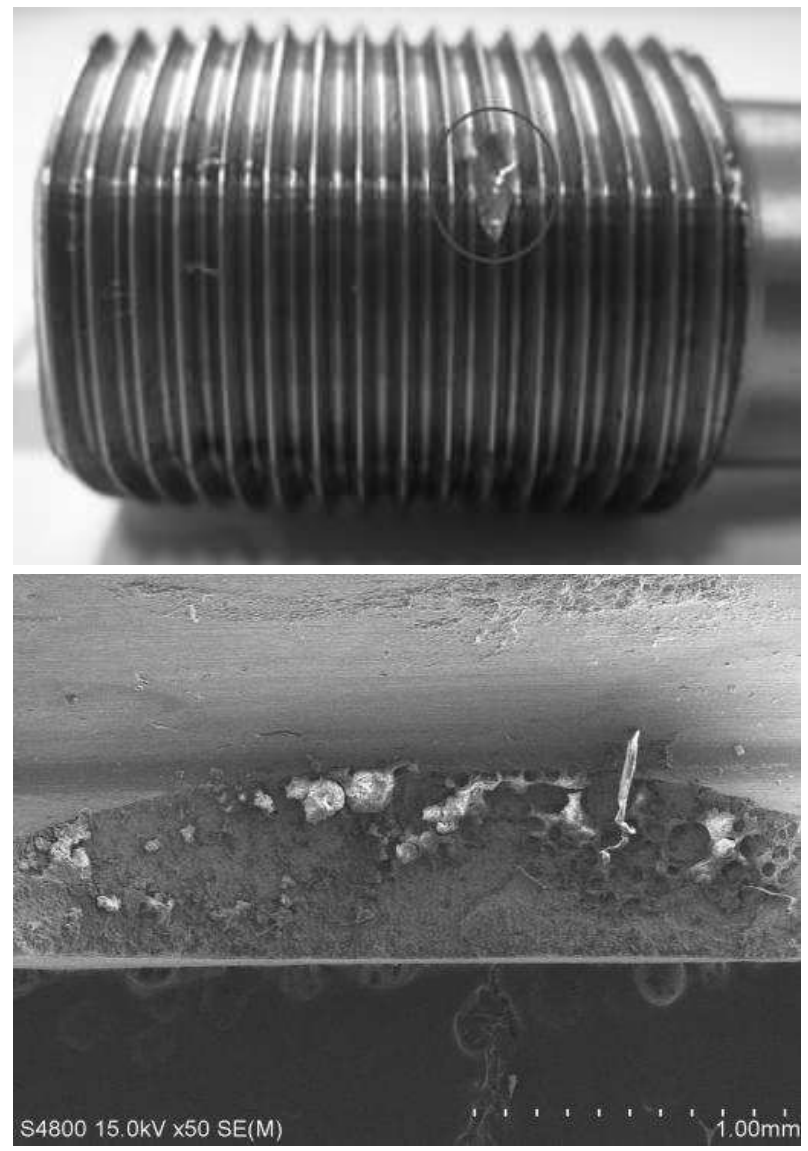

b) Seriously wearing status and partial enlarged drawing of thread forming tap
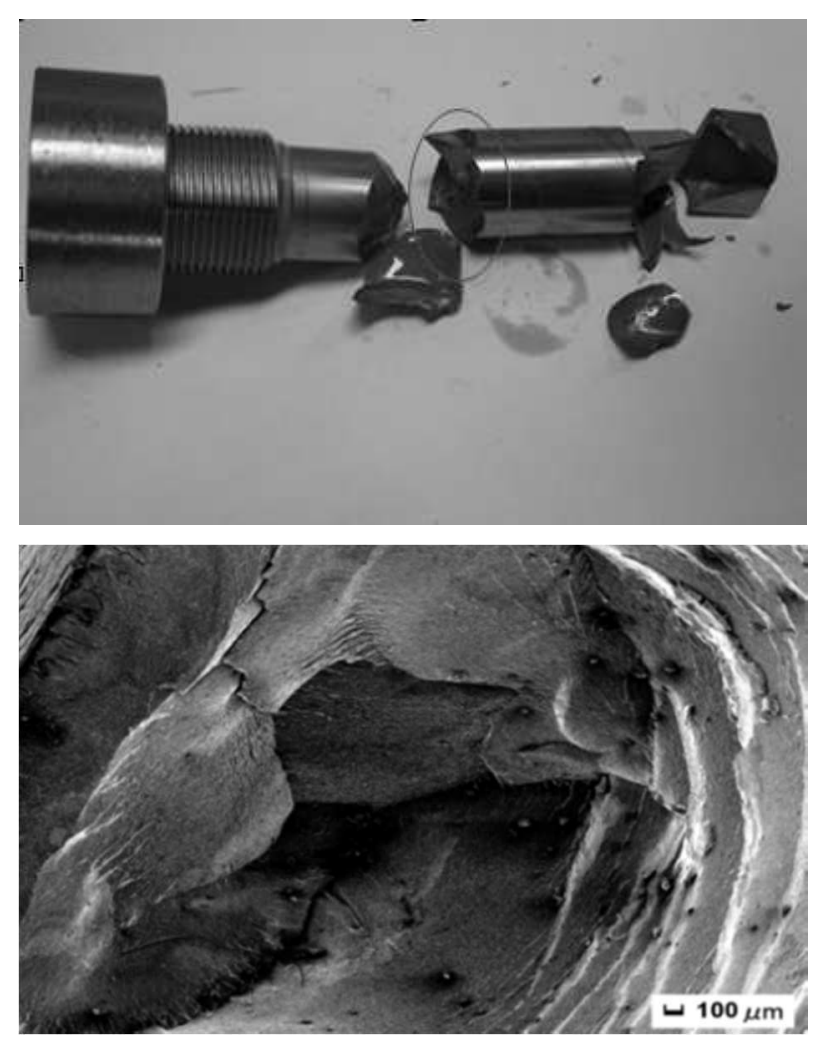

c) Broken status and partial enlarged drawing of thread forming tap

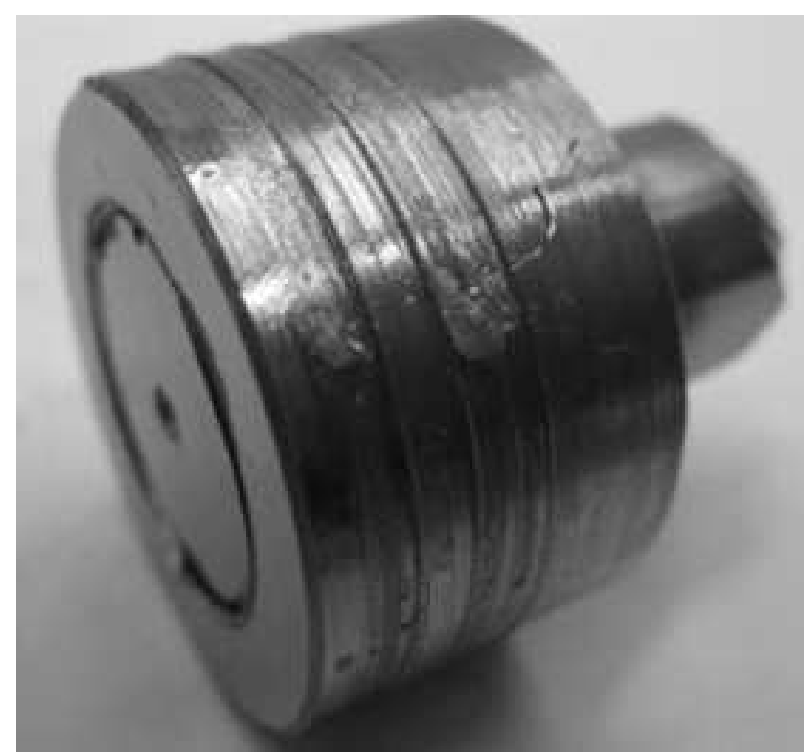

d) Slipping status of work pieces

Fig. 13 photos and partial micrograms of thread forming tap under different working process status

Fig. 13 shows separately the photos and partial micrograms of thread forming tap under wearing status, serious wearing status, broken status and slipping status.

\section{Conclusion}

Online monitoring system of cold extrusion of internal thread process is designed by the virtual instrument technology. LabVIEW8.5 is used as platform to realize the functions of data collection, data storage, artificial 
neural networks, real-time display of images, real-time monitoring of current status and recurrence of historical data; based on the foundation of the time-domain analysis and time-frequency-domain analysis of the torque signal, temperature signal, vibration signal, acoustic emission signal and transmitted signal, multi-sensors data fusion technology with the collaboration of multiple sensors is used to improve the monitoring precision; this online monitoring system can forecast processing status accurately, raise real-time alarm to replace or re-install the thread forming tap, accordingly avoids effectively the wreckage of thread forming tap and the scrap of work pieces. Thus the practical model of online monitoring system for cold extrusion of internal thread process can be used to monitor working process, it is valuable to popularize to the industry.

\section{Acknowledgment}

This project is supported by Open Foundation of Key Laboratory of Modern Agricultural Equipment, Ministry of Agriculture, P.R. China (Grant No. 201604003), Jiangsu Science and Technology Plan Project of China (Grant No. BE2015113), Natural Science Foundation of the Jiangsu Higher Education Institutions of China (Grant No. BKJB460016), the Agricultural Science \& Technology Independent Innovation Funds in Jiangsu Province of China (Grant No. CX(15)1047), National Natural Science Foundation (51672241), Project funded by China Postdoctoral Science Foundation (Grant No. 2016M600447) and the 14th batch High-level Talents Project for "Six Talents Peak" (Grant No. XCL092)

\section{Refrences}

[1] MIAO Hong, ZUO Dunwen, WANG Hongfeng, et al. (2010). Effect on the Thread Connection of Aircraft Landing Gear by the Impact Load. Journal of Vibration and Shock, Vol. 2, No.29, pp. 208-212. CNKI. China.

[2] XU Jiuhua, WANG Min, JIN Wenlin, et al. (1993). Study on Cold Form Tapping of Internal Threads of Superhigh Strength Steels. Acta Aeronautica Et Astronautica Sinica, Vol. 10, No.14, pp. 557-559. CNKI. China.

[3] XU Xiaojing, ZHANG Xuefeng, LIU Guilin, et al. (2008). Work-piece length of the effect on stress and analysis of cracking-criterion under equal channel angular pressing. Chinese Journal of Mechanical Engineering, Vol.1, No.44, 223-226. CNKI. China.

[4] WANG Min. (1998). Principle and Technology of Anti-Fatigue Manufacture. Jiangsu Science And Technology Publishing House, Beijing.

[5] HONG MIAO, QING MEI, JINGYUN YUAN, et al. (2017). Numerical Simulation and Experimental Research on Cold Form Tapping Process of Internal Thread. Manufacturing Technology, Vol. 17, No. 4, pp. 519-526. Engineering Village. USA.

[6] JIANG Lianmin, HU Xianghong. (2007). Analysis on the Surface Cracks of High Performance Q460/Z35 Plate. Wide and Heavy Plate, Vol.1, No.13, pp. 19-23. CNKI. China.

[7] XU Chunguang, WANG Xinyi, XIAO Dingguo. (1994). A New Method for the On-Line Monitoring of Tool State. Journal of Beijing Institute of Technology, No.2, pp. 48-20. CNKI. China.

[8] ZHENG Jianming, LI Yan. (2006). A New Wavelet Fractal Dimension and Its Application in Drill Wear Monitoring. Journal of Xi An University of Technology, Vol.22, No.2, pp. 128-131. CNKI. China.

[9] YANG Z.J., TAN Q.C., SHI E.J.. (2004). On-line monitoring of drilling torques of micro-drills. Journal of Engineering manufacture proceedings part B, Vol.3, No.218, pp. 1735-1740. SAGE Journals. USA.

[10] LEE L.Y., TARNG Y.S.. (2000). Drill fracture detection by the discrete wavelet transform. Journal of Materials Processing Technology, Vol.1, No.99, pp. 250-254. ELSEVIER. Netherlands.

[11] HIEBERT S.F., CHINNAM R.B.. (2000). Role of artificial neural networks and wavelets in on-line reliability monitoring of physical systems. Proceedings of the International Joint Conference on Neural Networks, Vol.6, pp. 369-374. IEEE computer society. USA.

[12] JIROUTOVA, D. (2016). Methodology of Experimental Analysis of Long-term Monitoring ofSandwich Composite Structure by Fibre-optic Strain Gauges. Manufacturing Technology, Vol. 16, No. 3, pp. 512-518. Engineering Village. USA. 